\title{
La race NDama dans le cheptel bovin du Sénégal
}

\author{
K.H.M KANH ${ }^{1,2,3^{*}}$, D.P SOKOURI ${ }^{1}$, M. DIOP ${ }^{2}$ et A. DIENG ${ }^{3}$ \\ ${ }^{1}$ Université Felix Houphouët Boigny d'Abidjan, Laboratoire de Génétique, UFR Biosciences, 22 B.P. 582 \\ Abidjan22, Côte d'Tvoire. \\ ${ }^{2}$ Isra-Lnerv, BP 2057, Dakar-Hann, Sénégal. \\ ${ }^{3}$ Université de Thiès, Ecole Nationale Supérieure d'Agriculture, Département Productions Animales, $\mathrm{km} 3$ \\ route de Khombol, BP A296 Thiès, Sénégal. \\ *Auteur correspondant ; E-mail : kanhmichael@yahoo.fr
}

\section{RESUME}

La présente étude fait une compilation des connaissances sur la race NDama au Sénégal. Cette race trypanotolérante se rencontre en Casamance (Sud) et au Sénégal oriental qui sont des zones infestées de glossine. La race NDama se subdivise en 3 sous populations et se déclinent sous diverses colorations du pelage, avec une prédominance du fauve. Le dimorphisme sexuel est assez marqué. Le poids moyen à l'âge adulte est de $300 \mathrm{~kg}$ pour les mâles contre $230 \mathrm{~kg}$ pour les femelles. La hauteur au garrot est de 105,6 $\pm 8,7 \mathrm{~cm}$ chez les mâles contre $102,0 \pm 4,7 \mathrm{~cm}$ chez les femelles. La puberté est atteinte à 27 mois et la durée de la gestation est respectivement de $285 \pm 2$ jours et $292 \pm 5$ jours lors de portées multiples. L'âge au premier vêlage est de 39,8 $\pm 0,8$ mois. La production laitière est médiocre et elle varie entre 0,1 et 1,75 litre par jour. Le taurin NDama a de bonnes aptitudes bouchères, avec un GMQ de 318,5 $\pm 60,23 \mathrm{~g}$ en élevage en stabulation. La race NDama est élevée selon différents modes de conduite d'élevage et son élevage se heurte à de nombreuses contraintes. Cependant, les perspectives de développement et de valorisation de cette race sont nombreuses.

(C) 2019 International Formulae Group. All rights reserved.

Mots clés: Taurin, trypanotolérance, aptitudes laitières et bouchères.

\section{The NDama breed in Senegal's cattle herd}

\begin{abstract}
This study summarizes the whole of knowledge about the NDama breed in Senegal. This trypanotolerant race is found in Casamance and eastern Senegal, which are tsetse-infested areas. The NDama breed is subdivided into 3 subpopulations and diclinates under various colors of coat with a predominance of the fawn color. Sexual dimorphism is very marked. The average weight in adult age is $300 \mathrm{~kg}$ for males against $230 \mathrm{~kg}$ for females. The height at the withers is $105.6 \pm 8.7 \mathrm{~cm}$ in males against $102.0 \pm 4.7 \mathrm{~cm}$ in females. Puberty is reached at 27 months and the duration of gestation is $285 \pm 2$ days and $292 \pm 5$ days, respectively, during multiple litters. Age of first calving is $39.8 \pm 0.8$ months. Milk production is very low and it varies between 0.1 and 1.75 liters per day. The NDama breed has good beefing abilities with a GMQ of $318.5 \pm 60.23$ $\mathrm{g}$ in breeding. NDama is raised according to different methods of breeding and its breeding faces many constraints. However, there are numerous prospects for development and valuing of this breed.
\end{abstract}

(C) 2019 International Formulae Group. All rights reserved

Keywords: Taurine breed, trypanotolerance, dairy production, beefing abilities. 


\section{INTRODUCTION}

La race bovine NDama constitue avec les races Lagunaire, Somba et Baoulé le rameau des taurins originaires d'Afrique de l'Ouest. Son berceau est le Fouta Djallon en Guinée. Ce sont des bovins trypanotolérants (Touré,1977), bien adaptés à leur milieu naturel, car elle vit et se reproduit dans ces milieux infestés par la mouche tsé-tsé (Okouyi, 2000). En Afrique de l'Ouest, seuls les taurins, dont fait partie la race bovine NDama possèdent cette particularité biologique qui est absente chez les zébus.

Au Sénégal, la race NDama constitue l'essentiel du cheptel bovin du sud, zone infestée de glossines, vecteur de la trypanosomose animale africaine (TAA). Cependant, la pression accrue (feu de brousse, déboisement abusif) sur les ressources naturelles, l'extension des champs de culture due à la croissance démographique, favorisent l'incursion des Zébus, trypanosensibles, et leur croisement avec la race Ndama. Ce fait constitue une menace potentielle de la pureté génétique de la race (ILRI, 2010). Cette situation fait craindre la réduction drastique des effectifs des animaux de race NDama et aussi la perte de la particularité biologique, la trypanotolérance, du fait des croisements avec les races sahéliennes. Cet état de fait a justifié la création du PROGEBE (Projet régional de gestion durable du bétail ruminant endémique) au Sénégal, au Mali, en Guinée et en Gambie. Ce projet a été conçu pour préserver et renforcer, de manière durable, les caractéristiques génétiques de trois espèces de bétail ruminant endémique (bovins de race NDama, ovins de race Djallonké et chèvre naine d'Afrique de l'Ouest), d'accroitre leur productivité et d'améliorer leur exploitation dans le cadre d'un environnement physique et institutionnel favorable (Sissokho et al., 2010).

L'effectif des bovins NDama était de 636240 têtes en 1993 (Sow et al., 1997). La population NDama a subi une croissance jusqu'à atteindre 1100000 têtes en 2004 . Puis, il s'en est suivi une décroissance jusqu'à nos jours. Des statistiques récentes situent le nombre de têtes de bovins Ndama entre 733 422 et 850000 (DAD-IS, 2016).

Cette étude tente de résumer l'ensemble des études et connaissances acquises sur la Ndama au Sénégal et ailleurs tout en mettant en valeur le potentiel de cette race et les contraintes liés à son élevage.

\section{LES RACES BOVINES DU SENEGAL}

$\mathrm{Au}$ Sénégal, les races bovines rencontrées peuvent être classés en 2 grands groupes, notamment qui sont les races locales et les races exotiques (Photo 1 et 2).

\section{Les races locales \\ Les races zébus}

Les zébus sont très bien adaptés aux zones sèches où ils résistent mieux aux stress thermiques. Leur zone d'extension coïncide avec la bande sahélienne comprise entre les isohyètes 200 et $600 \mathrm{~mm}$ (Missohou et Adakal, 2004). La couleur de la robe du zébu est très variable. Une multitude de couleurs allant du blanc au noir en passant par toutes les nuances de fauve et de noir est observée (Sokouri et al., 2007). Il existe au Sénégal deux (2) races locales de zébu, le zébu maure et le zébu Gobra (Photo 1). Le zébu maure est élevé par les Maures dans le Sahel mauritanien. Il est rencontré au Sénégal dans le Nord du pays au niveau de la vallée du fleuve Sénégal (Cissé, 1992). Quant au zébu Gobra, il se rencontre dans le Ferlo, le bassin arachidier et dans plusieurs autres endroits du pays où ils sont répandus dans de petits troupeaux (Mine, 1981).

\section{Les races taurines}

Les taurins sont localisés au sud du pays, en Casamance et dans la région de Kédougou. Il s'agit essentiellement de bovins NDama subdivisés en plusieurs types dont l'appellation peut varier d'une localité et/ou d'une communauté à une autre (Photo 1). L'on distingue ainsi la NDama grande, la NDama petite, la NDama moyenne et la NDama Fula aussi connue sous le nom de Gabu ou Fula. (DAD-IS, 2016). Elle descendrait directement du taurin Africain à 
longues cornes, présent au Sahara dès 4000 ans av. J.C (Meyer, 2017).

\section{La race métisse Djakoré}

Il existe aussi une race métisse locale plus ou moins stabilisée nommée Djakoré (Photo 1). Elle est aussi connue sous les noms de Djokore, Race du Sine, Bambara, taurin du Kaarta, Mande (DAGRIS, 2017). La race Djakoré résulte du croisement ancien entre le Gobra situé au Ferlo et le NDama trypanotolérant fixé en Casamance et dans la partie Sud du Sénégal oriental (Buldgen et Compère, 1984). Le bovin Djakoré se rencontre dans les provinces du Sine-Saloum, le Sénégal oriental et particulièrement dans les provinces sérères du Cayor et du N'Diambour (DAGRIS, 2017).

\section{Les races exotiques}

Les races exotiques sont généralement des animaux à haut rendement, importés pour la production de lait et dans une moindre mesure la production de viande. Pour les races laitières, il s'agit de la Montbéliarde, la Holstein, la Jersiaise, la Brune des Alpes et la Normande. La race Guzera est également rencontrée et très appréciée des éleveurs à cause de son aptitude bouchère. Elle est d'origine indienne de l'Etat du Gujarat et a été introduite au Sénégal à partir du Brésil en 1964 (Denis et Gauchet, 1978). D'autres races telles que les Gir, Girolando et Nelore sont également rencontrées (Photo 2). Elles sont importées du Brésil et sont utilisées dans les élevages modernes pour la production de viande. Les races exotiques se rencontrent dans les fermes privées généralement autour des grandes agglomérations ou elles sont utilisées pour la production de lait, et dans une moindre mesure la production de viande. Malgré leur adaptation relativement difficile au Sénégal, toutes ces races étrangères ont une bonne production laitière et des conformations comparées aux races locales (Njong, 2006)

\section{ORIGINE ET REPARTITION GEOGRAPHIQUE DU CHEPTEL NDAMA AU SENEGAL Origine}

La race NDama est localisée au Sénégal dans le Sud et le Sud-Est du pays. Il s'agit pour l'essentiel de bovins NDama subdivisés en plusieurs types. Elle descendrait directement du taurin Africain à longues cornes, présent au Sahara dès 4000 an av. J.C (Meyer, 2017). L'on a pensé longtemps que son centre d'origine était le Moyen-Orient, à partir duquel elle aurait été introduite en Afrique à une époque située entre 5000 et 2 350 avant Jésus-Christ. Elle aurait suivi une route passant par l'Egypte, le littoral méditerranéen jusqu'au Maroc, puis, descendant vers le sud, elle aurait rejoint son centre de dispersion actuel, le Massif du Fouta Djalon en Guinée. A partir de là, elle se serait progressivement répandue dans les régions limitrophes (Coulomb,1976). Cependant, cette théorie communément admise est maintenant battue en brèche. Des recherches récentes en génétique moléculaire (Hanotte et al., 2002) ont en effet révélé que les bovins africains les plus anciens (Bos taurus) sont originaires d'une région unique, située en Afrique. Cette découverte corrobore les résultats archéologiques qui établiraient la présence en Afrique d'un centre de domestication bovine (MacDonald et MacDonald, 2000). Ce centre de domestication serait situé au Sahara, et s'étendrait selon ces auteurs du sud libyen et $\mathrm{du}$ nord-ouest du Niger jusqu'au sud de l'Egypte. En outre, dans cette région, selon Magnavita (2006), n'était élevé que du bétail sans bosse, caractéristique de la Nubie ancienne jusqu'à une époque récente.

Le nom NDama ne provient pas du Wolof (petit) comme on aurait pu le penser, mais de la région de Kadé (Guinée) d'où la race s'est propagée un peu partout en Haute Guinée, au Sénégal (Diaite,1980). Le nom NDama serait donc tiré du dialecte pulaar (langue des Peulhs) et signifierait nain. De nos jours, la race NDama se retrouve dans tous les pays d'endémies trypanosomiennes d'Afrique de l'Ouest et du Centre, et même en Angola. 


\section{Répartition géographique du cheptel NDama au Sénégal}

L'aire de répartition des bovins NDama se situe dans les régions administratives de Ziguinchor, de Sédhiou, de Kolda, de Kédougou et de Tambacounda où ces animaux constituent l'essentiel du cheptel bovin de ces zones (Figure 1). En raison de sa rusticité et de sa trypanotolérance, le taurin NDama a su s'imposer dans cette zone hostile aux zébus et autres races exotiques. En Casamance, au Sénégal oriental et en Gambie, environ $30 \%$ des effectifs totaux des bovins NDama d'Afrique y sont élevés (Fall et al., 1982).

Selon ILRI (2010), si l'hypothèse selon laquelle la presque totalité du cheptel rencontré dans les régions de Kédougou, de Kolda et de Ziguinchor relevant de type bétail ruminant endémique bovins est admise, alors il est possible d'estimer celui-ci à 764960 têtes pour les bovins NDama au Sénégal.

\section{DESCRIPTION DES PARAMETRES MORPHO BIOMETRIQUES ET ZOOTECHNIQUES}

\section{Caractéristiques morphobiométriques}

Animal de petite taille, le bovin NDama est de type rectiligne, médioligne, et eumétrique. C'est un animal relativement compact et trapus, surtout chez les mâles. Chez les femelles, les formes sont harmonieuses et d'une grande finesse. Le profil facial est droit. La tête est large et forte. Le cornage assez variable allant de moyen à long avec des formes allant de la coupe à la lyre en passant par le croissant et la roue (Baldé, 2017). Les cornes sont aussi effilées à l'extrémité. Il existe aussi des individus sans cornes. Les cornes sont généralement ambrées avec des extrémités noires. Les muqueuses sont de couleurs variables (Diouf, 1991). Si elles sont claires chez la NDama classique, les animaux à muqueuses noires sont fréquents (Coulomb, 1976; Diouf, 1991). Les poils sont courts (Diouf, 1991). La NDama porte une robe généralement fauve, mais aussi de couleur variable, souvent unie allant du noir au froment en passant par diverses nuances de brun et de fauve (Diadhiou, 2001). Les robes blanches et gris clair qui feraient penser à un métissage plus ou moins lointain avec les zébus Gobra sont très fréquentes. Cependant, la robe fauve typique de la race NDama est la plus rependue avec $49.1 \%$ des robes rencontrées (Diack; 2009). D'autres robes sont assez courantes notamment celles de couleur grise, gris foncée et les robes marquées de noir comme les robes noires, gris-moucheté, pie-noir (Gueye et al., 1976). Ces dernières présentes surtout en Basse Casamance seraient dues, selon SATEC (1973), à une imprégnation de taurins trypanotolérants autre que la NDama. Des robes atypiques ne sont pas rares. Des individus à robes très foncées pouvant aller jusqu'au noir franc, pie noir, pie fauve peuvent également être rencontrés. Les Couleur de la robe chez les taurins N'Dama dans la zone d'emprise du CRZ de Kolda en haute Casamance sont diverses selon (Gueye et al., 1981). Avec une prédominance des robes froment suivit de la robe blanche ou gris clair et la robe gris foncé représentant respectivement $26,6 \pm 2,6 \%, 22,3 \pm 2,6 \%$ et $12,4 \pm 2 \%$ des effectifs de bovins de la zone.

La peau est fine et souple. Le fanon modérément développé est surtout apparent chez le taureau. Les membres sont fins, les sabots petits et durs. Le dimorphisme sexuel est marqué. Le taureau est épais et trapu, d'allure assez lourde avec une encolure courte et puissante. La femelle est plus fine et d'allure légère, avec la mamelle modeste et les trayons fins. Le tempérament est éveillé et ces animaux s'adaptent rapidement aux bons comme aux mauvais traitements (Diouf, 1991). Selon Diack (2009) et Diouf et al. (2017), il existe trois variétés de la race NDama au Sénégal : la NDama-grande, la NDama moyenne et la NDama-petite avec des hauteurs au garrot respectives de $108,7 \mathrm{~cm}$, $104,5 \mathrm{~cm}$ et $100,7 \mathrm{~cm}$. La variété NDamagrande comporte de nombreux phénotypes se 
traduisant surtout par des colorations de la robe (souvent claire) et des muqueuses. Ces animaux semblent très proches du Gambia dwarf ou Gambia short horn. Cependant, la majorité des sujets présentent de longues cornes disposées en lyre, très caractéristiques chez le taurin NDama. La variété NDamapetite, quant à elle, est de format plus réduit. La robe froment est la plus fréquente. Elle représente la NDama classique de type guinéen (Diouf, 1991). La variété NDama moyenne représente un type intermédiaire entre la grande NDama et la petite.

Les mensurations corporelles de la race NDama sont consignées dans le Tableau 1. Ces mensurations sont moyennes. Cependant, elles sont supérieures à celles des autres taurins trypanotolérants, notamment la race Baoulé et la race Lagunaire (Soro et al., 2015 ; AU-BIAR, 2019). Le poids moyen chez les sujets adultes varie entre 280 à $350 \mathrm{~kg}$ chez le mâle et de 250 à $300 \mathrm{~kg}$ pour les femelles (Meyer, 2017).

\section{. Performances zootechniques - Performances de reproduction \\ $\checkmark$ Chez la femelle}

Une étude menée en milieu paysan sur les performances de reproduction des races bovines locales de Côte d'Ivoire, a donnée l'intervalle de vêlage moyen chez la race Ndama de 474,10 $\pm 147,81$ quand elle était de $495 \pm 16$ au Sénégal (Tableau 2). Le taux moyen de fécondité et le taux moyen d'avortement ont été respectivement de $83,20 \%$ et de 3,05\% (Sokouri et al., 2010). Tous ces paramètres se sont avérés plus intéressants chez les bovins NDama que chez les autres races locales (Baoulé et zébus).

Les premières chaleurs apparaîtraient vers l'âge de 15 mois chez les bovins Ndama du Congo et chez les génisses N'Dama élevées au Centre Régional Zootechniques de Minankro (Bouaké) et à la ferme de I'IRCT de Bouaké ( Ralambofiringa, 1978; Akouango et al., 2010). La puberté serait atteinte à 27 mois ( Ralambofiringa, 1978) voire 28 mois
(Gyawu et al., 1986) pour un poids moyen estimé à $175 \pm 20 \mathrm{~kg}$ en Côte d'Ivoire (Meyer et Yesso, 1990) voire $176 \pm 24 \mathrm{~kg}$ au Ghana. Pour d'autres auteurs, la puberté apparaît entre 26 (781 \pm 156 jours) et 31 mois soit $949 \pm 79$ jours (Osei et al., 1991). Une mise à la reproduction à l'âge de 27 mois $(779 \pm 119$ jours) a été recommandée par Akouango et al. (2010). L'influence de la saison de la naissance sur l'âge à la puberté a été signalée par plusieurs auteurs (Gyawu et al., 1986; Akouango et al., 2010). Les génisses nées durant la saison pluvieuse sont plus précocement pubères que celles nées en saison sèche (Gyawu et al., 1986; Akouango et al., 2010). La durée de la gestation serait respectivement de $285 \pm 2$ jours (Coulomb, 1976) voire $292 \pm 5$ jours lors de portées multiples en Guinée (Kamga-Waladjo et al., 2006). L'âge au premier vêlage a été mesuré au CRZ (Centre de Recherche Zootechnique) de Kolda au Sénégal à 39,8 $\pm 0,8$ mois avec un coefficient de variation de $14,1 \%$ et un intervalle inter-vêlages de $495 \pm 16$ (Fall et al., 1982).

\section{$\checkmark$ Chez les mâles}

L'âge à la puberté des taureaux NDama est de $510 \pm 19$ jours avec un poids de $153,5 \pm 21 \mathrm{~kg}$. Le volume moyen d'un éjaculat NDama est de 2,25 $\pm 0,43 \mathrm{ml}$. Le sperme de bonne qualité a une motilité massale de 2,68 \pm 0,75 et une motilité individuelle de 2,74 \pm 0,65 . La concentration en spermatozoïdes est de $0,743 \pm 0,179\left(10^{9} / \mathrm{ml}\right)$, et le nombre total de spermatozoïdes est de $1,485 \pm 0,340\left(10^{9}\right.$ spermatozoïdes $/ \mathrm{ml}$ ) pour un volume de l'éjaculat de 4,05 ml (Tamboura et al., 1992).

\section{Performances de production}

\section{$\checkmark \quad$ Aptitudes bouchères}

Le bovin NDama est surtout élevé pour ses qualités bouchères. Les performances de production bouchère des bovins NDama sont liées à l'environnement d'élevage. Elles peuvent ainsi varier d'un pays à un autre et d'un système d'exploitation à un autre. Son poids à la naissance varie entre 13 et $19 \mathrm{~kg}$ (Fall et al., 1982 ; Sissokho et al., 2010 ; 
N'Goran et al., 2016). Cette race présente des prédispositions à la production de viande; le rendement carcasse et de l'ordre de 50 à $55 \%$ pour des sujets pouvant atteindre $300 \mathrm{~kg}$ de poids vif (N'Diaye, 1990). Le poids moyen adulte des mâles NDama est de $370 \mathrm{~kg}$ et 250 kg pour les femelles en Guinée, contre $300 \mathrm{~kg}$ pour les mâles et $250 \mathrm{~kg}$ pour les femelles au Sénégal. Le dimorphisme sexuel est très marqué; les mâles étant plus grands que les femelles (Diao 1991). La croissance pondérale des mâles est assez lente chez les taurins NDama. Des mesures effectuées sur des taurillons présélectionnés et mis en stabulation au CRZ de Kolda au Sénégal ont donné un GMQ de $318,5 \pm 60,23 \mathrm{~g}$ et un poids moyen de $216 \pm 28,83 \mathrm{Kg}$ (Mbaye et al., 1985). Des données collectées par Coulomb (1976) en Côte d'Ivoire ont permis d'observer une croissance relativement lente avec des GMQ (gain moyen quotidien) plus élevés en début de croissance (de 0 à 7 mois). De 8 à 45 mois, une baisse en général des GMQ est constatée (Tableau 3).

\section{$\checkmark$ Production laitière}

$\mathrm{La}$ production laitière de la vache NDama est médiocre, voire négligeable (Coulomb, 1976; Okouyi et al., 2014). La durée de lactation relevé par Pagot (1985) est de 300 jours (Tableau 4). La moyenne de lait journalière par vache est de $1,96 \mathrm{~kg}$ de lait par jour dans la ferme d'élevage de l'Okpara du Bénin (Gbangboche et Alkoiret, 2011) et de 2,7 kg par jour pendant les 4 premiers mois d'allaitement au CRZ de Bouaké en Côte d'Ivoire (Coulomb, 1976). Au centre zootechnique de Kolda au Sénégal ces valeurs sont de 0,8055 $\pm 0,3858$ litres/vache/jour avec des productions variant de 0,1 à 1,75 litres pour des animaux de la station du CRZ de Kolda (Sissokho et al., 2012). Des études conduites en Casamance au Sénégal, dans l'objectif d'identifier des vaches exceptionnelles dans les troupeaux villageois ont permis d'enregistrer une production moyenne de 1,37 $\pm 0,45$ litres par jour avec quelques individus ayant une production qui varie entre 2,5 et 3 litres par jour (Sissokho, 2011).

\section{GESTION DES BOVINS NDAMA}

\section{Système d'élevage des bovins NDama}

Au Sénégal, les ressources végétales disponibles, qui constituent la base de l'alimentation, déterminent les différents modes de conduite des troupeaux. Les systèmes d'élevage sont rarement spécialisés (Duteurtre, 2006).

\section{$\checkmark$ Système transhumant}

Les bovins NDama sont exploités en petits troupeaux de 5 à 100 têtes effectuant quelquefois de courtes transhumances en saison sèche vers les régions côtières plus basses et mieux arrosées. Ce système d'exploitation est pratiqué dans le berceau de la race à savoir, le Fouta Djallon, en Guinée (Drame, 1994). Au Sénégal, les éleveurs ne pratiquent pas de transhumance, car les animaux vivent dans la zone la plus humide du pays.

\section{$\checkmark \quad$ Le système traditionnel agropastoral}

Le système agropastoral se fonde sur l'association de l'élevage aux cultures pluviales (mil, arachide, coton, etc.) et irriguées (riz, tomate, oignon, etc.). Ce système se rencontre principalement dans le bassin arachidier, la vallée du fleuve Sénégal et la zone Sud (de la Casamance au Sud-Est du pays). Ce système d'élevage est pratiqué chez $67 \%$ des bovins et $62 \%$ des petits ruminants (Habimana, 2008).

\section{$\checkmark$ Le système traditionnel pastoral}

L'élevage des bovins NDama se fait généralement en milieu traditionnel sous forme sédentaire. Pendant la saison des cultures, les animaux pâturent dans les jachères et les zones non cultivées. En saison sèche, les animaux sont ramenés dans les zones de culture où ils consomment les résidus de récolte (Munyaneza, 2013). C'est le système le plus fréquent au Sénégal.

$\checkmark$ Système de "ranching"

Dans les systèmes de "ranching" principalement développés au Zaïre, au 
Congo, en Côte d'Ivoire et au Nigéria, les troupeaux NDama sont exploités uniquement pour la boucherie; les femelles ne sont pas traites. Il en résulte une meilleure croissance des jeunes et une amélioration du format mais aussi une diminution de la docilité (Drame, 1994). Ce système n'est pas encore pratiqué au Sénégal sur les bovins NDama.

\section{Les contraintes à l'élevage des bovins NDama}

Les contraintes à l'élevage des bovins NDama sont les mêmes que celles rencontrées dans le secteur de l'élevage tant au Sénégal que dans toute la sous-région. Elles sont de différents ordres.

\section{Les contraintes alimentaires}

La sous-alimentation revêt un caractère endémique en zone tropicale surtout lorsqu'elle est associée à une difficulté d'abreuvement. Cette sous-alimentation est surtout liée à la rareté et à la pauvreté des pâturages en saison sèche au Sénégal (Nishimwe, 2008). Le cheptel de bovin NDama fait face, à certains endroits, à la diminution des pâturages et surtout à la raréfaction des points d'abreuvement pendant la saison sèche. L'eau est une contrainte majeure à la bonne conduite de l'élevage dans la zone. Sa saisonnalité fait que pendant la saison sèche chaude, la quête d'un point d'eau reste une préoccupation pour les éleveurs (Bâ, 1992).

\section{Les contraintes pathologiques}

Les contraintes sanitaires sont beaucoup plus fréquentes dans les élevages traditionnels. On note la présence de glossines, vecteurs de la trypanosomose animale africaine (TAA), dans le Sud du Sénégal, dont l'incidence mensuelle de la trypanosomose de $7 \%$ pour les animaux utilisés pour la traction animale (Faye et al., 1996).

L'on note la persistance de certaines maladies comme la pasteurellose et les maladies telluriques telles les charbons bactéridien et symptomatique.de plus, l'absence des vétérinaires privés (installés dans les centres urbains) dans les zones de l'étude fait que les tradipraticiens sont très consultés et les connaissances indigènes très utilisées. Aussi, ceci explique le fait que l'automédication soit une pratique courante surtout dans les zones peu accessibles (Baldé, 2017). La contrainte majeure reste cependant, la forte mortalité des veaux, en rapport direct avec leur infestation par les parasites gastrointestinaux: Ascaridia, Trichostrongylus, Paramphistum (Ba, 1992; Diao, 1991). Bien que Les parasitoses (hémoparasitoses, ectoparasitoses et parasitoses gastrointestinales) bien que très courantes ne sont pas considérées par les agropasteurs comme une menace pour les bovins Ndama (Baldé, 2017) cette perception a été rapportée par Claxton et Leperre (1991), Mattioli et al. (1993) et Agymang et al. (1997) dans les élevages villageois de Gambie.

\section{Les contraintes liées à la faible productivité de la race}

Les contraintes de productivité sont dues aux faibles performances des races locales. Chez le taurin Ndama, le poids d'un animal de 4 ans varie entre 270 et $320 \mathrm{~kg}$ alors qu'il peut atteindre $492 \mathrm{~kg}$ chez les bovins Gobra en station (Sow et al., 1991) et varie de 300 à $500 \mathrm{~kg}$ pour les zébu Azawak adultes (Adjou, 2006; Oumarou, 2004). En outre, l'on note une faiblesse du potentiel laitier des races locales dont la production oscille entre 1 et 3 litres de lait par jour avec une période de lactation de 180 jours (Nishimwe, 2008). Ces valeurs sont inférieures à celle du Zébu Azawak qui oscille entre 2 et 8 litres en milieu paysan et 10 litres en station pour des durées de lactation respectives de 6 et 1 mois (Vias, 2013; Siddo, 2017). Par ailleurs, un manque de formation des éleveurs et un faible niveau de technicité sont observés pour le système pastoral extensif (Kabera, 1978).

Les contraintes structurelles et économiques

Au Sénégal comme dans la plupart des pays africains, il existe une défaillance du système d'encadrement des éleveurs. Rares 
sont les pays africains où l'intensification des productions animales est une priorité (Nishimwe, 2008). En outre, le crédit agricole est difficilement accessible avec des taux d'intérêt très élevés (Amahoro, 2005). Il faut aussi noter que l'Etat a adopté l'intensification de la production laitière par le biais des biotechnologies notamment l'insémination artificielle (Diop, 1993). Cependant, les taux de réussite restent faibles comparativement au taux de référence de l'insémination artificielle qui est de 60-70\% (Kouamo, 2006). Ces performances sont dues à un non-respect strict des protocoles d'IA par les inséminateurs et les éleveurs. Cela se matérialise par une flexibilité sur le choix des vaches à inséminer, le non-respect du calendrier de travail et de la bonne conduite des animaux sélectionnés avant et après insémination (Habimana, 2012). Ces croisements peuvent, s'ils ne sont pas contrôlés, constituer un moyen d'introgression de nouveaux gènes et surtout la perte de la "pureté" génétique au sein du cheptel NDama et de sa singularité biologique qu'est la trypanotolérance.

\section{PERSPECTIVES}

Les taurins NDama peuvent être utilisés à plusieurs niveaux. Pour la productivité de la vache au Sénégal, seuls la viande et le lait sont analysés. Les autres types de productions tels que le fumier, la traction, les cuirs et peaux, bien que non négligeables sont considérés comme faisant partie des avantages généralement non quantifiés. Cette situation ouvre alors en plus du domaine de l'amélioration, de nombreuses perspectives d'études et d'action pouvant permettre de découvrir et valoriser le potentiel non exploré de la race NDama (Nesseim, 1995).

\section{Amélioration génétique}

Les perspectives d'amélioration
génétique chez la race NDama sont

prometteuses, car les caractéristiques des taurins NDama les rendent aptes à plusieurs axes d'amélioration. Les bovins NDama peuvent être utilisés dans le cadre de croisements avec différentes races laitières ou à viande dans le but de créer de nouvelles races. Ces races peuvent être adaptées à l'environnement et aux réalités locales tout en combinant les caractères de rusticité de la race NDama et ceux de production des races exotiques. La race NDama pourrait aussi faire l'objet d'études plus approfondies afin de découvrir tout son potentiel génétique afin de définir des programmes de sélection et de valorisation adaptés au contexte socio culturel et économique des éleveurs et milieux d'élevage de cette race.

\section{Traction animale}

La race NDama est utilisée pour la culture attelée, car ce bovin présente une bonne aptitude à la traction. Le bœuf NDama bien dressé peut être utilisé comme animal de trait en culture attelée; il pourrait tirer une charge de $350 \mathrm{~kg}$ à la vitesse de $4 \mathrm{~km} / \mathrm{h}$ (Coulomb, 1976). Aussi, la formation des éleveurs aux bénéfices du lisier, du purin et du fumier peut permettre de substituer l'engrais chimique à l'engrais biologique et réduire par voie de conséquence les coûts de certains intrants.

\section{Produits dérivés}

Le cuir des taurins NDama est fin, de bonne qualité et connu sous le nom de « vachette de Guinée ». Il est utilisé pour la fabrication de sacoches, de sacs ou de chaussures (Pagot, 1985; Okouyi, 2000). Cette activité peut s'avérer prometteuse au Sénégal, dans la mesure où $22 \%$ des 4772 tonnes de peau et cuir importés dans ce pays sont d'origine bovine (MEPA, 2016). En outre, les cornages peuvent faire l'objet de valorisations à travers des œuvres d'art et des accessoires. 

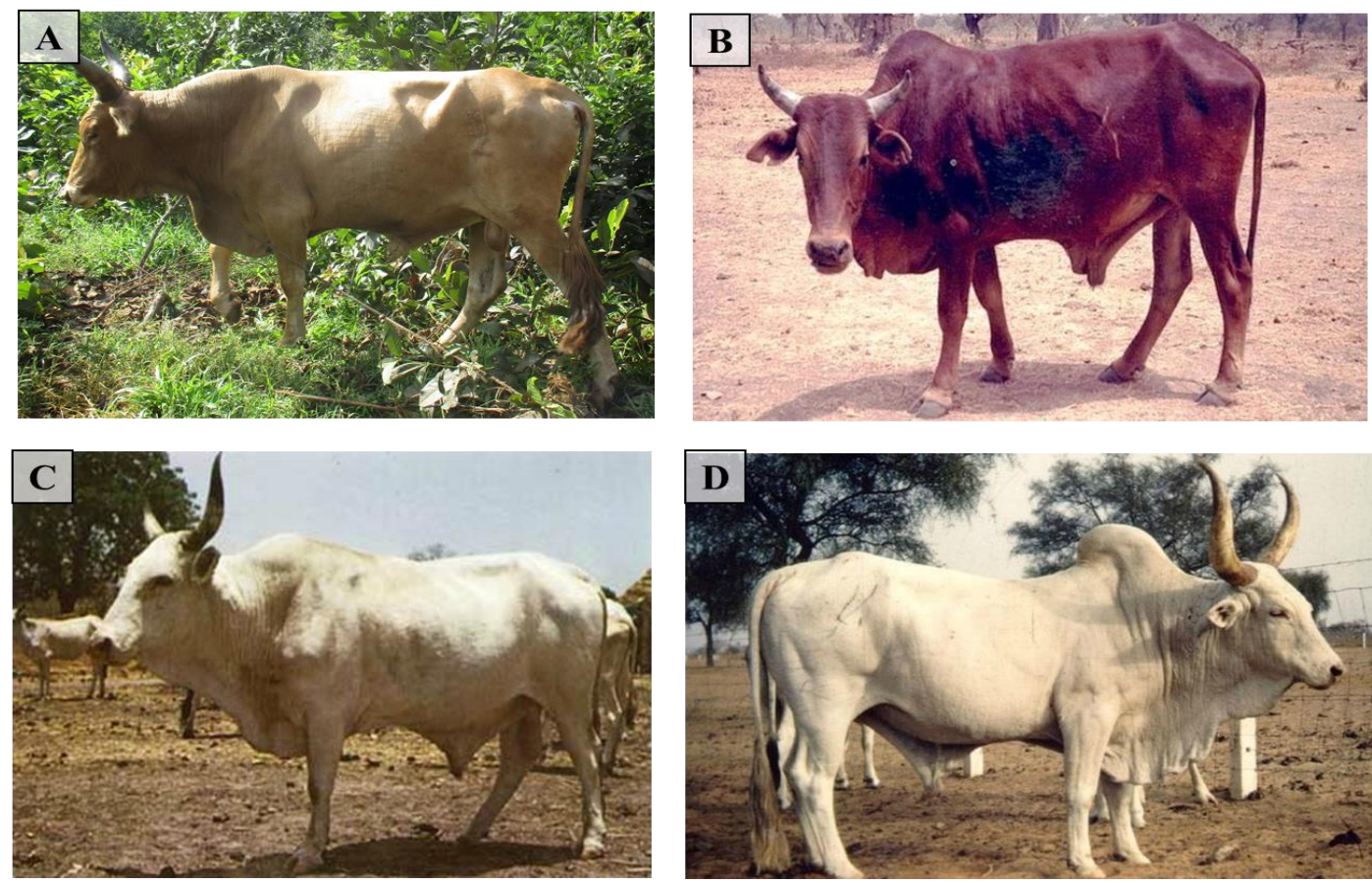

Photo1: Races locales au Sénégal (a: N’dama, b: zébu Maure (Meyer, 2017), c:zébu Djakoré(Meyer, 2015), d:zébu Gobra(Meyer, 2017)).
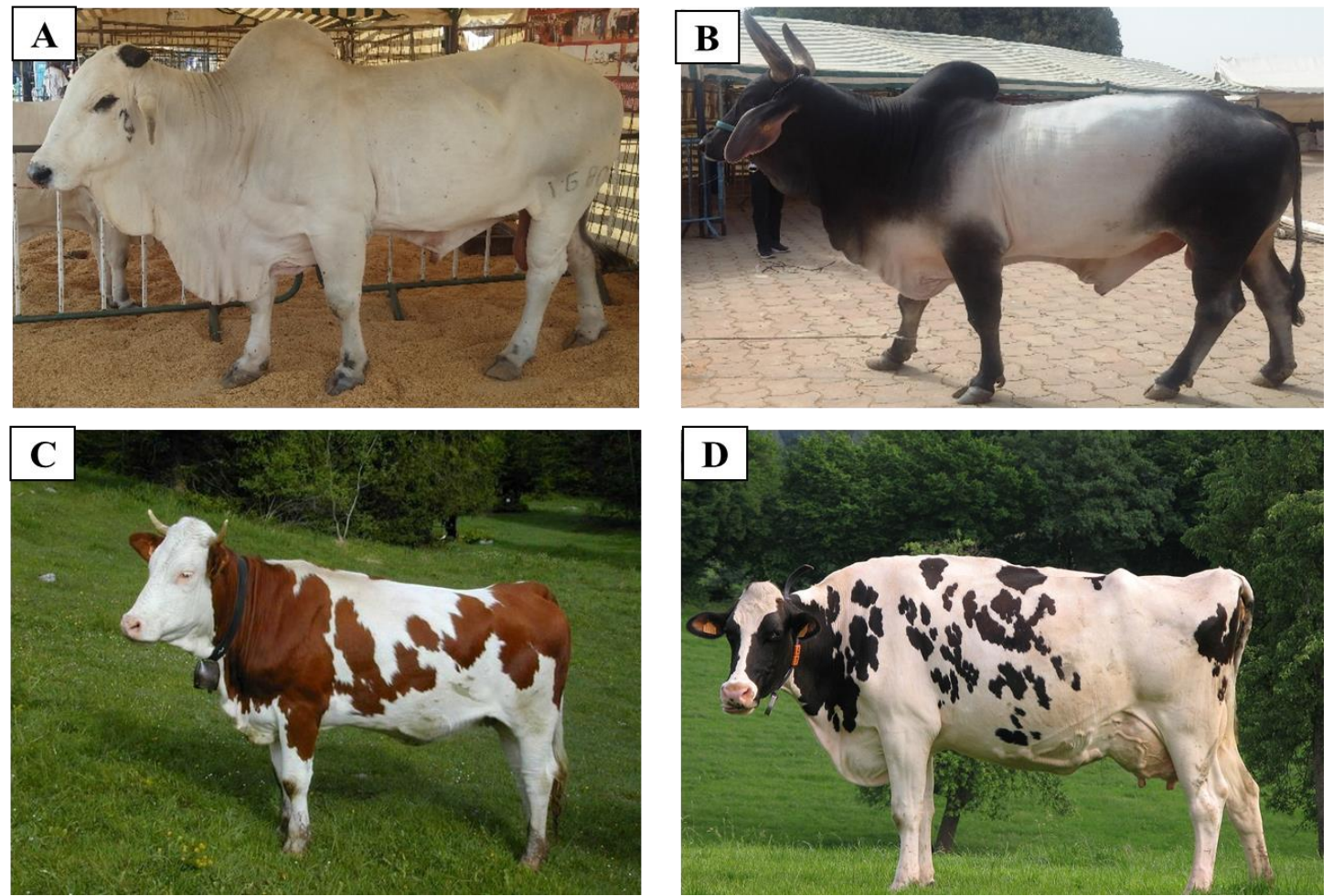

Photo 1: Races exotiques au Sénégal (A: Taureau Nelore, B : Taureau Guzerat, C: Vaches Montbéliard (Meyer, 2019), D : Vache Holstein(Meyer, 2019)). 


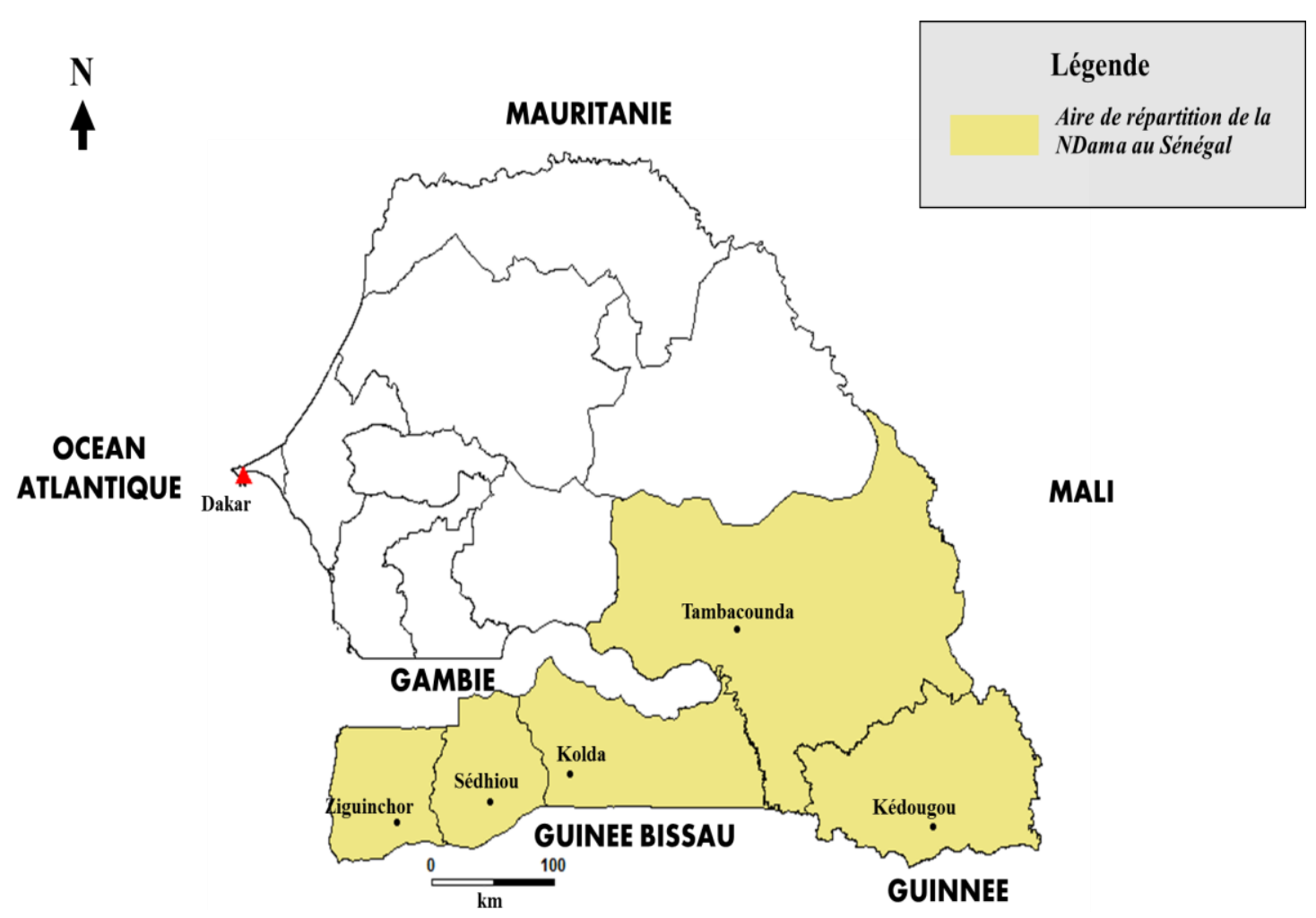

Figure 1: Aire de répartition de la Ndama au Sénégal.

Tableau 1: Mensurations moyennes corporelles des taurins NDama adultes élevés en milieu paysan au Sénégal.

\begin{tabular}{lll}
\hline \multicolumn{1}{c}{ Paramètres } & Mâles & Femelles \\
\hline Hauteur au garrot en (cm) & $105,6 \pm 8,7(12)$ & $102,0 \pm 4,7(208)$ \\
Profondeur du thorax (cm) & $56,4 \pm 1,44(12)$ & $53,7 \pm 8,7(208)$ \\
Périmètre du thorax (cm) & $151,4 \pm 6,5(12)$ & $139,2 \pm 6,7(208)$ \\
Longueur du corps (cm) & $120,3 \pm 8,9(12)$ & $111,5 \pm 10,2(30)$ \\
Longueur de la tête (cm) & $34,6 \pm 1,84(12)$ & $37,5 \pm 2,49(208)$ \\
Longueur du crâne (cm) & $12,30 \pm 0,69(12)$ & $14,20 \pm 0,92(208)$ \\
Circonférence du museau (cm) & $28,6 \pm 1,23(12)$ & $31 \pm 3,21(208)$ \\
Longueur de la queue (cm) & $83,1 \pm 7,2(12)$ & $77,2 \pm 6,7(208)$ \\
\hline $\begin{array}{l}\text { Source : Baldé (2017) } \\
\text { () : Nombre d'animaux mesuré. }\end{array}$ & &
\end{tabular}


Tableau 2: Quelques paramètres zootechniques moyens des NDama en fonction des pays.

\begin{tabular}{lcccc}
\hline & Sénégal & Guinée & Côte d'Ivoire & Nigeria \\
\hline Poids naissances mâle (Kg) & 18 & 17,5 & 19 & 18,1 \\
Poids naissances femelle (Kg) & 17 & 16,8 & 17 & 15,1 \\
Poids adulte mâle (Kg) & 300 & 370 & 225 & $n c$ \\
Poids adulte femelle (Kg) & 230 & 250 & 235 & 275 \\
Age au premier vêlage (mois) & 33,19 & 36 & $33,20 \pm 5,64$ & $22,8 \pm 1,18$ \\
Intervalle vêlage -vêlage & $495 \pm 16$ & $420-534$ & $474,10 \pm 147,81$ & $363 \pm 31,16$ \\
(jours) & & & & \\
\hline
\end{tabular}

Source: Diallo (2017); DAD-IS (2015); Fall et al. (1982); N'Goran et al. (2016); Roberts et Gray (1973a).

nc : non connus

Tableau 3: Evolution des poids moyens des taurins NDama mâles Source : Coulomb (1976).

\begin{tabular}{|c|c|c|c|c|c|c|c|c|}
\hline Age & $\begin{array}{c}\text { Poids } \\
\text { (kg) }\end{array}$ & $\begin{array}{c}\text { G.M.Q } \\
\text { (g) }\end{array}$ & Age & $\begin{array}{c}\text { Poids } \\
\text { (kg) }\end{array}$ & $\begin{array}{c}\text { G.M.Q } \\
\text { (g) }\end{array}$ & Age & $\begin{array}{c}\text { Poids } \\
\text { (kg) }\end{array}$ & $\begin{array}{c}\text { G.M.Q } \\
\text { (g) }\end{array}$ \\
\hline Nais & 17,7 & 629 & 4 mois & 66,2 & 333 & 17 mois. & 165 & 387 \\
\hline $1 \mathrm{sem}$. & 22,1 & 500 & 4,5 mois & 71,2 & 407 & 18 mois. & 176,6 & 397 \\
\hline 2 sem. & 25,6 & 429 & 5 mois & 77,3 & 427 & 19 mois & 187,9 & 237 \\
\hline 3 sem. & 28,6 & 386 & 5,5 mois & 83,7 & 381 & 20 mois & 195,0 & 319 \\
\hline 4 sem. & 31,3 & 343 & 6 mois & 89,8 & 413 & 21 mois & 204,9 & 257 \\
\hline 5 sem. & 33,7 & 414 & 7 mois & 102,2 & 210 & 22 mois & 212,6 & 235 \\
\hline 6 sem. & 36,6 & 357 & 8 mois & 108,7 & 203 & 23 mois & 219,9 & 250 \\
\hline 7 sem. & 39,1 & 386 & 9 mois & 114,8 & 132 & 24 mois. & 227,4 & 208 \\
\hline 8 sem. & 41,8 & 371 & 10 mois & 118,9 & 193 & 27 mois & 249,4 & 254 \\
\hline 9 sem. & 44,4 & 386 & 11 mois & 124,7 & 161 & 30 mois & 269,5 & 281 \\
\hline $10 \mathrm{sem}$. & 47.1 & 357 & 12 mois & 129,7 & 97 & 33 mois & 295,1 & 177 \\
\hline $11 \mathrm{sem}$. & 49,6 & 429 & 13 mois & 132,6 & 226 & 36 mois & 231,2 & 138 \\
\hline $12 \mathrm{sem}$. & 52,6 & 357 & 14 mois & 139,6 & 203 & 39 mois & 323,6 & 52 \\
\hline $\begin{array}{l}3 \text { mois } \\
3,5 \text { mois. }\end{array}$ & $\begin{array}{l}55,1 \\
60,2\end{array}$ & 340 & $\begin{array}{l}15 \text { mois } \\
16 \text { mois }\end{array}$ & $\begin{array}{l}145,7 \\
157,2\end{array}$ & 383 & $\begin{array}{l}42 \text { mois } \\
45 \text { mois }\end{array}$ & $\begin{array}{l}328,5 \\
335,6\end{array}$ & 78 \\
\hline
\end{tabular}

GMQ : Gain Moyen Quotidien. 
Tableau 4 : Production laitière chez la vache NDama.

\begin{tabular}{cccc}
\hline Année & $\begin{array}{c}\text { Production moyenne par } \\
\text { lactation }(\mathbf{k g})\end{array}$ & $\begin{array}{c}\text { Durée de la } \\
\text { lactation }(\mathbf{j})\end{array}$ & Effectifs \\
\hline 1953 & 421,67 & 300 & 40 \\
1954 & 488,6 & 300 & 40 \\
1955 & 481 & 300 & 47 \\
1957 & 686 & 300 & 47 \\
1958 & 658,73 & 300 & 47 \\
\hline
\end{tabular}

Source : Pagot (1985).

\section{Conclusion}

La race NDama occupe une place importante dans le cheptel bovin sénégalais. Au-delà de son petit gabarit qui pourrait constituer le prétexte pour la délaisser, elle demeure une race bovine bien adaptée au milieu naturel infesté de glossines et de parasites divers. Quoique les différentes tentatives d'amélioration n'aient pas atteint les résultats escomptés, il convient de pousser la réflexion sur cette ressource génétique. Car, au regard de ses potentialités avérées et de son importance économique pour les ménages ruraux, il conviendrait de définir des axes de recherche et de développement pour la valorisation de cette ressource génétique transfrontalière.

\section{CONFLIT D'INTERETS}

Les auteurs déclarent qu'ils n'ont aucun conflit d'intérêts.

\section{CONTRIBUTIONS DES AUTEURS}

KHMK: Recherche documentaire et rédaction de l'article de synthèse. DPS : Contribution à la recherche documentaire, corrections, amendements de l'article et validation de l'article après sa rédaction. MD: Contribution à la recherche documentaire, corrections et amendements de l'article. AD: supervision générale de la rédaction de l'article de synthèse corrections et amendements de la synthèse.

\section{REMERCIEMENTS}

Les auteurs remercient le Projet régional de gestion durable du bétail ruminant endémique (PROGEBE) pour la documentation, le projet (HAAGRIM) pour le financement de la mobilité au Sénégal. L'Université de Thiès (Sénégal) à travers l'école National Supérieur d'Agriculture (ENSA) et l'Institut Sénégalais de Recherches Agricoles (ISRA) pour l'accueil, le cadre d'étude et la documentation.

\section{REFERENCES}

Adjou MPFA. 2006. Evaluation Des Performances Zootechniques Des Bovins de Race Borgou En Sélection à La Ferme d'Elevage de l'Okpara (Benin). Thèse de Doctorat en Médecine Vétérinaire, Ecole Inter-Etats des Sciences et Médecine Vétérinaires de Dakar, Université Cheikh Anta Diop de Dakar, Dakar, Sénégal.

Agyemang K, Dwinger RH, Little DA, Rowlands GJ. 1997. Village Ndama cattle production in West Africa. Six years of research in the Gambia, Nairobi, International livestock, Research institute and Banjul, International Trypanotolerance Centre, p. 131. 
Akouango F, Ngokaka C, Ewomango P, Kimbembe E. 2010. Caractérisation morphométrique et reproductive des taureaux et vaches NDama du Congo. Anim. Genet. Resour. Info., 46: 41-47.

Amahoro E. 2005. Contribution à l'étude du profil métabolique chez des vaches laitières dans les fermes laitières intensives périurbaines de Dakar (cas des fermes de Wayembam et de Niacoulrab). Thèse de Doctorat en Médecine Vétérinaire, Ecole Inter-Etats des Sciences et Médecine Vétérinaires de Dakar, Université Cheikh Anta Diop de Dakar, Sénégal, p. 115.

AU-BIAR. 2019. La Lagunaire une race bovine en voie de disparition. AU-BIAR. http://www.auibar.org/component/jdownloads/viewdo wnload/129-kya/2890-20170209-kyalagune-dwarf-shorthorn-fr, Consulté le 06/07/19 à 11 heures.

Bâ O. 1992. Contribution à l'étude du système de production laitière de la vache NDama (Bos Taurus) en haute Casamance : contraintes et stratégies d'amélioration. Thèse de Doctorat en Médecine Vétérinaire, Ecole Inter-Etats des Sciences et Médecine Vétérinaires de Dakar, Université Cheikh Anta Diop de Dakar, Sénégal, p. 93.

Baldé AT. 2017. Caractérisation phénotypique du taurin NDama du Sénégal. Mémoire de Master, Université Cheikh Anta Diop, Sénégal, p. 58.

Buldgen A, Compère R. 1984. Caractéristiques des troupeaux villageois des bovins Djakorés sénégalais (Sénégal Oriental). Tropicultura, 2: 10-15. http://www.tropicultura.org/ content/v2n1.html

Cissé M. 1992. Situation actuelle de la production laitière au Sénégal. Rapport ISRA, LNERV/BP, $\mathrm{p} 13$.

Claxton J, Leperre P. 1991. Parasite burdens and host susceptibility of Zebu and Ndama cattle in village herds in the Gambie. Veterinary Parasitology, 40(3-
4): $\quad 293-304$.

4017(91)90109-9

Coulomb J. 1976. La race NDama : quelques caractéristiques zootechniques. Rev. Elev. Méd. Vét. Pays Trop., 29: 367-380. http://revues.cirad.fr/index.php/REMVT/ article/view/8005

DAD-IS. 2016. Domestic Animal Diversity Information System. DAD-IS. http://dad.fao.org/

DAGRIS. 2017. Domestic Animal Genetic Resources Information System (International Livestock Research Institute).

DAGRIS. http://sen.dagris.info/fr/node/2428/

Denis JP, Gauchet D. 1978. Le cheptel bovin au Sénégal. Synthèse des résultats. LNERV/ISRA, P. 57.

Diack F. 2009. Etude des systèmes d'élevage et caractérisation morphobiométrique du taurin NDama du sud du Sénégal. Mémoire de DEA. Université Cheikh Anta Diop, Dakar, Sénégal, p. 58.

Diadhiou A. 2001. Etude comparative de deux moyens de maitrise de la reproduction (L'implant CRESTAR ${ }^{\circledR}$ et la Spirale PRID ${ }^{\circledR)}$ chez les vaches NDama et Cobra au Sénégal. Thèse de Doctorat en Médecine Vétérinaire, Ecole Inter-Etats des Sciences et Médecine Vétérinaires de Dakar, Université Cheikh Anta Diop de Dakar, Sénégal, p. 84.

Diallo H. 2017. N'dama Guinée. http://dad.faoorg/fr/home.htm

Diao B. 1960. Caractéristiques du système agro-pastoral de haute-Casamance, l'exemple de la zone de Kolda. Thèse de Doctorat en Médecine Vétérinaire, Ecole Inter-Etats des Sciences et Médecine Vétérinaires de Dakar, Université Cheikh Anta Diop de Dakar, Sénégal, p. 84.

Diaite A. 1980. Contribution à l'étude des bovins trypanotolérants de la HauteCasamance. Thèse de Doctorat en Médecine Vétérinaire, Ecole Inter-Etats des Sciences et Médecine Vétérinaires de Dakar, Université Cheikh Anta Diop de Dakar, Sénégal, p. 83. 
Diop PEH. 1993. Biotechnologie et élevage africain. In Maîtrise de la Reproduction et Amélioration Génétique des Ruminants. Les Nouvelles Editions Africaines du Sénégal : Dakar, Sénégal ; 145-150.

Diouf MN. 1991. Endocrinologie sexuelle chez la femelle NDama au Sénégal. Thèse de Doctorat de Médecine Vétérinaire. Thèse de Doctorat en Médecine Vétérinaire, Ecole Inter-Etats des Sciences et Médecine Vétérinaires de Dakar, Université Cheikh Anta Diop de Dakar, Sénégal, p. 121.

Diouf MN, Seck MT, Thevenon S, Diop M, Sow RS, Seck MM, Sissoko M, Diop M, Skilton R, Kemp S, Njahira M, Kyallo M, Wanjala B, Mbanjo G, Kaduma E, Nzuki I, Wamonje F, Ndila M et Kemp S. 2017. Taurin NDama au Sénégal : diversité de trois sous-populations. ISRA, p.5.

Drame EHD. 1994. Cinétique hormonale (œstrogène, progestérone et LH) chez la femelle NDama superovulée. Thèse de Doctorat en Médecine Vétérinaire, Ecole Inter-Etats des Sciences et Médecine Vétérinaires de Dakar, Université Cheikh Anta Diop de Dakar, Sénégal, p .146.

Duteurtre V. 2006. Etats des lieux de la filière lait et des produits laitiers au Sénégal. Sénégal, Dakar, p. 94.

Fall A, Diop M, Sandford J, Wissocq Y, Durkin J, Trail JC, Gueye E. 1982. Evaluation des productivités des ovins Djallonke et des taurins NDama au Centre de Recherches zootechniques de Kolda (Sénégal). Rapport de recherche $\mathrm{n}^{\circ}$ 3, CIPEA, Addis-Abeba, Ethiopie, p. 74.

Faye A, Diack A, Dieye PN, Fall A. 1996. Projet de recherche sur les inters relations entre les pathologies parasitaires des bovins de trait en zone subhumide du Sénégal. Rapport d'avancement. CRZ/Isra, Kolda, Sénégal, p.10.

Gbangboche AB, Alkoiret, TI, 2011. Reproduction et production de lait des bovins de race Borgou et NDama au Bénin. J. Appli. Biosci., 46 : 3185-3194.

Gueye E, Nicolas A, Diao B. 1976. Etudes de la composition de la structure et de la couleur des robes des troupeaux taurins. CRZK/ ISRA, Kolda, Sénégal. p. 26.

Gueye E, Nicolas A, Diao B. 1981. Couleur de La Robe Chez Les N'Dama de Haute Casamance, Sénégal. Rev. Elev. Méd. Vét. Pays Trop., 34(3): 275-279. https://agritrop.cirad.fr/455099/1/ID4550 99.pdf

Gyawu P, Osei SA, Karikari PK, Karteng FA, Asaré K. 1986. Use of radioimmunoassay to monitorreproductive performance of indigenous cattle in the humid forestzone of Ghana. In: Second workshop on the reproduction of thetrypanotolerant livestock in west and central africa. Banjul, Gambia ; p. 32.

Habimana R. 2012. Evaluation de La Qualité Des Services D'insémination Artificielle Bovine Au Sénégal. Cas Des Régions de Kaolack et Kolda. Thèse de Doctorat en Médecine Vétérinaire, Ecole Inter-Etats des Sciences et Médecine Vétérinaires de Dakar, Université Cheikh Anta Diop de Dakar, Sénégal, p.86.

Habimana S. 2008. Evaluation de la séroprévalence et impact des maladies abortives sur la réussite de l'Insémination artificielle bovine au Sénégal. Cas de la région de Thiès. Thèse de Doctorat en Médecine Vétérinaire, Ecole Inter-Etats des Sciences et Médecine Vétérinaires de Dakar, Université Cheikh Anta Diop de Dakar, Sénégal, p. 82.

Hanotte O, Bradley DG, Ochieng JV, Verjee Y, Hill W, Rege JEO. 2002. African pastoralism: genetic imprints of origins and migrations. Science, 296: 336-339. DOI: $10.1126 /$ science. 1069878

ILRI. 2010. Gestion durable du bétail ruminant endémique en Afrique de l'Ouest (PROGEBE) Etude de référence, Sénégal. Rapport ILRI, Nairobi, p. 108. 
Kabera F. 1978. Contribution à l'amélioration du taux de réussite de l'insémination artificielle bovine dans les campagnes d'insémination artificielle réalisées par le PAPEL au Sénégal. Thèse de Doctorat en Médecine Vétérinaire, Ecole InterEtats des Sciences et Médecine Vétérinaires de Dakar, Université Cheikh Anta Diop de Dakar, Sénégal, p. 101.

Kamga-Waladjo AR, Mbaïndingatoloum FM, Lapo R, Thiam O, Sultan J, Diop PEH. 2006. Caractéristiques de reproduction des NDama utilisées en insémination artificielle bovine en République de Guinée. Rev. Afr. Santé Prod. Anim., 4: 69-72.

https://orbi.uliege.be/bitstream/2268/168 755/1/2014\%20OKOUYI\%20reproducti on\%20Ndama\%20synthese.pdf

Kouamo J. 2006. Evaluation Technicoéconomique Des Stratégies D'insémination Artificielle En Zone Sylvo-pastorale: Cas de La Région de Louga. Thèse de Doctorat en Médecine Vétérinaire, Ecole Inter-Etats des Sciences et Médecine Vétérinaires de Dakar, Université Cheikh Anta Diop de Dakar, Sénégal, p.64.

MacDonald K, MacDonald RH. 2000. The origins and development of domesticated animals in arid West Africa. In The Origins and Development of African Livestock: Archaeology, Genetics, Linguistics, and Ethnography, Blench RM, MacDonald KC (Eds). UCL Press: London; 127-162.

Mattioli RC, Bah M, Faye J, Kora S, Gassama M. 1993. A comparison of field tick infestation Ndama, zebu an Ndama zebu crossbred cattle. Veterinary Parasitology, 47(1-2): 139-148. DOI: 10.1016/0304-4017(93)90184-o

Magnavita C. 2006. Ancient Humped Cattle in Africa: A View from the Chad Basin, African Archaeological Review, 23: 5584.

DOI : http://dx.doi.org/10.1007/s10437-0069008-z
Mbaye M, Cheikh MM, Malang B, Bineta B. 1985. Sélection bouchère du taurin NDama: Etude des performances pondérales et sexuelles chez les taurillons en contrôle individuel. ISRA, Kolda, Sénégal. p. 8.

MEPA. 2016. Rapport de Revue sectorielle 2016. Ministère de l'élevage et des productions animales, Dakar, Sénégal.

Meyer C. 2015. Dictionnaire des Sciences Animales. http://dico-sciencesanimales.cirad.fr/

Meyer C. 2017. Dictionnaire des Sciences Animales. http://dico-sciencesanimales.cirad.fr//

Meyer C. 2019. Dictionnaire des Sciences Animales. http://dico-sciencesanimales.cirad.fr/

Meyer C, Yesso P. 1991. Courbe de progestérone plasmatique du cycle ostral des races taurines trypanotolérantes de Cote d'Ivoire. Rev. Elev. Méd. Vét. Pays Trop., $\quad$ 44(2): 193-198. https://agritrop.cirad.fr/394043/1/ID3940 43.pdf.

Meyer C, Yesso P. 1990. Maîtrise de l'cestrus chez les Bovins (trypanotolérants) N'Dama et Baoulé. Maisons-Alfort, CIRAD/IEMVT : France ; 53-54.

Mine P. 1981. Aptitudes du zébu peulh sénégalais (Gobra) pour la production de viande. Thèse de Doctorat en Médecine Vétérinaire, Ecole Inter-Etats des Sciences et Médecine Vétérinaires de Dakar, Université Cheikh Anta Diop de Dakar, Sénégal, p. 66.

Missohou MA, Adakal EH. 2004. Situation actuelle et perspectives d'une gestion durable des ressources génétiques bovines d'Afrique de l'Ouest. Colloq. Int. Sur Ressources Génétiques, Ouagadougou, Burkina Faso, 167-173.

Munyaneza C. 2013. Evaluation de différentes doses de "PMSG utilisées lors de la synchronisation des chaleurs sur la fertilité des vaches de la race NDama. Mémoire de Master, Ecole Inter-Etats des Sciences et Médecine Vétérinaires de 
Dakar, Université Cheikh Anta Diop de Dakar, Sénégal, p. 32.

Nesseim DT. 1995. Introduction de la superovulation chez la femelle bovine NDama pendant la saison sèche au Sénégal. Thèse de Doctorat en Médecine Vétérinaire, Ecole Inter-Etats des Sciences et Médecine Vétérinaires de Dakar, Université Cheikh Anta Diop de Dakar, Sénégal, p. 93.

Nishimwe K. 2008. Evaluation des facteurs de variation du taux de réussite de l'insémination artificielle bovine en milieu traditionnel au Sénégal : cas de la région de Thiès. Thèse de Doctorat en Médecine Vétérinaire, Ecole Inter-Etats des Sciences et Médecine Vétérinaires de Dakar, Université Cheikh Anta Diop de Dakar, Sénégal, p. 85.

N'Goran KE, Zakpa LG, Dago DN, Lallié HDMN, Sokouri DP, Doumbia L. 2016. Production and Reproduction Parameters Analysis of NDama Cattle Breed in the Dairy Station of Yamoussoukro (SLY), in the Savannah Zone, in Côte d'Ivoire. World Journal of Research and Review, 3(6):

https://www.wjrr.org/download_data/WJ RR0306017.pdf

Njong P. 2006. Adaptation des vaches à haut potentiel de production laitière en milieu tropical : cas de bovins Holstein introduits en 2002 dans la ferme de Wayembam au Sénégal Thèse de Doctorat en Médecine Vétérinaire, Ecole Inter-Etats des Sciences et Médecine Vétérinaires de Dakar, Université Cheikh Anta Diop de Dakar, Sénégal, p.64.

Oumarou A. 2004. "Production laitière et croissance du zébu Azawak en milieu réel: suivi et évaluation technique à miparcours du projet d'appui à l'élevage des bovins de races Azawak en zone agropastorale au Niger Thèse de Doctorat en Médecine Vétérinaire, Ecole Inter-Etats des Sciences et Médecine Vétérinaires de Dakar, Université Cheikh Anta Diop de Dakar, Sénégal, p. 82.
Okouyi M, Kamga Waladjo AR, Diarra S, Hanzen C. 2014. Caractéristiques de reproduction de la femelle trypanotolérante de race NDama. Rev. Afr. Santé Prod. Anim., 12:3-7. DOI : https://orbi.uliege.be/handle/2268/16875 5

Okouyi MWM. 2000. Maitrise de la reproduction de la femelle NDama. Thèse de Doctorat en Médecine Vétérinaire, Ecole Inter-Etats des Sciences et Médecine Vétérinaires de Dakar, Université Cheikh Anta Diop de Dakar, Sénégal, p. 127.

Osei SA, Karikari PK, Tuah AK, Gyawu P, Opoku RS. 1991. The reproductive performance of indigenous beef cattle breeds raised on farm in Ghana. In: The Third workshop on the reproduction of the trypanotolerant livestock in the West and Central Africa. Banjul, Gambia; 1935 .

Pagot J. 1985. L'Elevage en Pays Tropicaux, Maisonnoeuve GP (ed). ACCT: Paris.

Ralambofiringa A. 1978. Note sur les manifestations du cycle oestral et sur la reproduction des femelles NDama (Côte d'Ivoire). Rev. Elev. Méd. Vét. Pays Trop., 31: 91-94. DOI : https://doi.org/10.19182/remvt.8184

SATEC, 1973. Etude et définition d'un programme d'intervention en faveur de l'élevage. Paris, France. p. 90.

Siddo S. 2017. Evaluation Socio-économique Du Potentiel de Diffusion Du Zébu Azawak Sélectionné Au Niger. Thèse de $\mathrm{PhD}$, Université de Liège, Belgique, p.124.

Sissokho MM. 2011. Rapport technique du quatrième trimestre octobre-décembre 2010, PROGEBE, p. 19.

Sissokho MM, Diatta A, Camara Y. 2010. Rapport technique annuel d'activité 2009. PROGEBE, p. 70.

Sissokho MM, Diokou A, Ndao S. 2012. Rapport Technique $\mathrm{N}^{\circ} 4$ OctobreDécembre 2011. PROGEBE, p. 16.

Sokouri DP, Loukou NE, Yapi-Gnaoré CV, Mondeil F, Gnangbe F. 2007. 
Caractérisation phénotypique des bovins à viande (Bos taurus et Bos indicus) au centre (Bouake) et au nord (Korhogo) de la Cote d'Ivoire. Anim. Genet. Resour. Inf., 40: 43-53. http://www.fao.org/tempref/docrep/fao/0 10/a1128t/a1128t04.pdf

Sokouri DP, Yapi-Gnaore CV, N'guetta ASP, Loukou NE, Kouao BJ, Toure G, Kouassi A, Sangaré A. 2010. Performances de reproduction des races bovines locales de Côte d'Ivoire. J. Appl. Biosci., 36: 2353-2359. http://m.elewa.org/JABS/2010/36/4.pdf

Soro B, Sokouri PD, Dayo GK, N'Guetta ASP, Yapi-Gnaoré CV. $2015 b$. Morphometric and physical characteristics of Baoulé cattle in the "Pays Lobi" of Côte d'Ivoire. Livestock Research for Rural Development, 27(7): 0121-3784.

http://www.lrrd.org/lrrd27/7/soko27124. html.

Sow RS, Diop M, Mbaye M. 1997. L'élevage au Sénégal : politiques de développement et gestion des ressources génétiques des animaux domestiques. ISRA/LNERV, p.7.
Sow RS, Denis JP, Trail JCM, Thiongane PI, Mbaye M. 1991. Note Sur La Barymétrie Comme Moyen de Sélection Indirecte Du Poids Vif Chez Le Zébu Gobra Au Sénégal. Rev. Elev. Méd. Vét. Pays Trop., $\quad \mathbf{4 4}(1)$ : 97-100. https://agritrop.cirad.fr/394025/1/ID3940 25.pdf

Tamboura H, Tahiri-Zagret C, Coulibaly M. 1992. Influence du climat tropical humide sur les caractéristiques spermatiques de races taurines en Côte d'Ivoire. Bull. Anim. Heal. Prod. Afr. Bull., 40:185-196.

Touré SM. 1977. La trypanotolérance. Revue de connaissances. Rev Elev Méd Vét Pays Trop, 30: 144-157. http://www.beep.ird.fr/collect/eismv/inde x/assoc/TD89-4.dir/TD89-4.pdf

Vias FSG. 2013. Etude Relative à La Formulation Du Programme D'actions Détaillé de Développement de La Filière Lait En Zone UEMOA. Rapport Niger, Niamey, p. 53. 